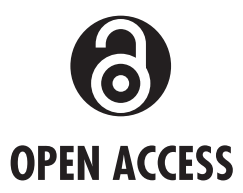

${ }^{1}$ Toronto Congenital Cardiac Center for Adults, Peter Munk Cardiac Center, University of Toronto, Toronto General Hospital, Toronto, Ontario, Canada

${ }^{2}$ Toronto Joint Department of Medical Imaging, University of Toronto, University Health Network, Toronto, Ontario, Canada

${ }^{3}$ Department of Diagnostic Imaging, The Hospital for Sick Children, Toronto, Ontario, Canada

${ }^{4}$ Division of Cardiology, The Hospital for Sick Children, Toronto, Ontario, Canada ${ }^{5}$ Division of Cardiovascular Surgery, The Hospital for Sick Children, Toronto, Ontario, Canada

\section{Correspondence to} Dr Rachel M Wald, Toronto Congenital Cardiac Center for Adults, Peter Munk Cardiac Center, University of Toronto, Toronto General Hospital, Toronto, ON M5G 2N2, Canada; Rachel.Wald@uhn.ca

PC and GRK contributed equally.

Received 3 February 2020 Revised 20 May 2020 Accepted 26 May 2020 Published Online First 6 July 2020

Check for updates

(C) Author(s) (or their employer(s)) 2021. Re-use permitted under CC BY-NC. No commercial re-use. See rights and permissions. Published by BMJ.

To cite: Charla P, Karur GR, Yamamura $\mathrm{K}$, et al. Heart 2021:107:142-149.

\title{
Augmentation of pulmonary blood flow and cardiac output by non-invasive external ventilation late after Fontan palliation
}

\author{
Pradeepkumar Charla, ${ }^{1}$ Gauri Rani Karur (D) , ${ }^{2}$ Kenichiro Yamamura (D) , 1 \\ Shi-Joon Yoo, ${ }^{3}$ John T Granton, ${ }^{1}$ Erwin N Oechslin, ${ }^{1}$ Ashish Shah, ${ }^{1}$ Leland N Benson, ${ }^{4}$ \\ Osami Honjo, ${ }^{5}$ Luc Mertens, ${ }^{4}$ Rafael Alonso-Gonzalez, ${ }^{1}$ Kate Hanneman, ${ }^{2}$ \\ Rachel M Wald ${ }^{1,4}$
}

\section{ABSTRACT}

Objectives Although a life-preserving surgery for children with single ventricle physiology, the Fontan palliation is associated with striking morbidity and mortality with advancing age. Our primary objective was to evaluate the impact of non-invasive, external, thoracoabdominal ventilation on pulmonary blood flow (PBF) and cardiac output (CO) as measured by cardiovascular magnetic resonance (CMR) imaging in adult Fontan subjects.

Methods Adults with a dominant left ventricle post-Fontan palliation (lateral tunnel or extracardiac connections) and healthy controls matched by sex and age were studied. We evaluated vascular flows using phase-contrast CMR imaging during unassisted breathing, negative pressure ventilation (NPV) and biphasic ventilation (BPV). Measurements were made within target vessels (aorta, pulmonary arteries, vena cavae and Fontan circuit) at baseline and during each ventilation mode.

Results Ten Fontan subjects ( $50 \%$ male, 24.5 years (IQR 20.8-34.0)) and 10 matched controls were studied. Changes in PBF and $\mathrm{CO}$, respectively, were greater following BPV as compared with NPV. In subjects during NPV, PBF increased by $8 \%\left(\Delta 0.20 \mathrm{~L} / \mathrm{min} / \mathrm{m}^{2}(0.10-0.53)\right.$, $p=0.011)$ while CO did not change significantly $(\triangle 0.17$ $\left.\mathrm{L} / \mathrm{min} / \mathrm{m}^{2}(-0.11-0.23), \mathrm{p}=0.432\right)$; during BPV, PBF increased by $25 \%\left(\Delta 0.61 \mathrm{~L} / \mathrm{min} / \mathrm{m}^{2}(0.20-0.84)\right.$, $\mathrm{p}=0.002)$ and CO increased by $16 \%\left(\Delta 0.47 \mathrm{~L} / \mathrm{min} / \mathrm{m}^{2}\right.$ $(0.21-0.71), p=0.010)$. Following $B P V$, change in PBF and $\mathrm{CO}$ were both significantly higher in subjects versus controls $\left(0.61 \mathrm{~L} / \mathrm{min} / \mathrm{m}^{2}(0.2-0.84)\right.$ vs $-0.27 \mathrm{~L} / \mathrm{min} / \mathrm{m}^{2}$ $(-0.55-0.13), p=0.001$; and $0.47 \mathrm{~L} / \mathrm{min} / \mathrm{m}^{2}(0.21-0.71)$ vs $0.07 \mathrm{~L} / \mathrm{min} / \mathrm{m}^{2}(-0.47-0.33), \mathrm{p}=0.034$, respectively). Conclusion External ventilation acutely augments PBF and $\mathrm{CO}$ in adult Fontan subjects. Confirmation of these findings in larger populations with longer duration of ventilation and extended follow-up will be required to determine sustainability of haemodynamic effects.

\section{INTRODUCTION}

Complex congenital heart disease consisting of a functional single ventricle is typically managed with a Fontan palliative strategy in early childhood, where the systemic venous return is directed to the pulmonary arteries in the absence of an interposed pumping chamber. ${ }^{12}$ Notwithstanding excellent surgical results in children, the natural history late after Fontan surgery is characterised by an inexorable increase in systemic venous pressure over time, relatively decreased pulmonary blood flow (PBF) and lower cardiac output (CO) as compared with patients with a biventricular circulation. With age, increasing numbers of Fontan survivors develop a constellation of findings attributable to vascular insufficiency (venous congestion and/or low output) described as failing Fontan physiology, which can result in morbidity and mortality due to intracardiac and extracardiac disease. ${ }^{3-5}$ Therapeutic options for those with failing Fontan physiology are limited: surgical strategies such as Fontan conversion or heart transplantation are high-risk endeavours deemed appropriate in a minority of patients, ${ }^{6}$ and medical therapies will only provide temporary, symptomatic relief. ${ }^{7}$

Systemic venous return in Fontan patients is significantly influenced by respiration. Positive pressure ventilation is best avoided or minimised in postoperative Fontan patients. Early studies have demonstrated a beneficial effect of non-invasive negative pressure ventilation (NPV) on $\mathrm{PBF}$ and $\mathrm{CO}$ in children after bidirectional and total cavopulmonary connections. ${ }^{8-11}$ The haemodynamic effects of NPV have not yet been described in an ambulatory population late after Fontan surgery. Therefore, our primary objective was to evaluate the acute effects of NPV on PBF and $\mathrm{CO}$ using a cardiovascular magnetic resonance (CMR) imaging compatible non-invasive external thoraco-abdominal ventilator with comparison to a novel biphasic ventilation (BPV) mode in adults with a Fontan palliation. Non-invasive, accurate and reproducible vascular flows measurements can be achieved using phase-contrast (PC) CMR analysis. ${ }^{12} 13$ We hypothesised that BPV and NPV modes, respectively, would augment both indexed PBF and CO. In addition to establishing feasibility and efficacy of non-invasive external ventilation for adult Fontan subjects in the outpatient setting, we also sought to determine safety and tolerability of differing ventilation strategies in this pilot study. 


\section{METHODS}

\section{Study population}

Adults with prior Fontan palliation (lateral tunnel or extracardiac connections) and a dominant left ventricle (LV) followed at Toronto General Hospital were eligible for inclusion if their supervising cardiologist determined that they were clinically stable and safe to undergo CMR. Subjects were excluded if they were known to have ongoing arrhythmia, $\geq$ moderate valve regurgitation or stenosis, LV ejection fraction $<40 \%$, Fontan pathway obstruction, persistent Fontan fenestration, venovenous collaterals (oxygen saturation $<90 \%$ ), chronic obstructive lung disease (forced expiratory volume in $1 \mathrm{~s} /$ forced vital capacity $<60 \%$ ), moderate obstructive sleep apnoea (apnoeahypopnoea index $>20$ ), obesity (body mass index $>35 \mathrm{~kg} / \mathrm{m}^{2}$ ) and/or chest wall deformities. Age-matched and sex-matched healthy controls were recruited for comparison. Patients and controls were directly approached for study inclusion and informed consent was obtained. This study did not include indirect patient involvement or the public. Each participant was contacted by study investigators at multiple time-points during and after the study to document feedback regarding safety and tolerability of the device and CMR protocol.

\section{External ventilation device}

A commercially available external ventilator (Hayek RTX, Medivent International, London, UK) was used for non-invasive thoraco-abdominal ventilation for each participant. Ventilation equipment consisted of a plastic cuirass shell for application over the anterior chest and upper abdomen, a computerised control unit and tubing to connect the two. Given the exploratory nature of this study, we sampled multiple ventilation settings and selected the respective settings for NPV and BPV associated with the greatest augmentation of flows as determined by CMR. The following ventilation modes were applied to each subject: $\mathrm{NPV}$, a continuous ventilation mode set at $-30 \mathrm{~cm} \mathrm{H}_{2} \mathrm{O}$, and $\mathrm{BPV}$, a biphasic mode alternating between negative and positive pressures set at $-21 /+7 \mathrm{~cm} \mathrm{H}_{2} \mathrm{O}$. All CMR measurements were made following $10 \mathrm{~min}$ of active ventilation and with ventilation ongoing; a $10 \mathrm{~min}$ 'washout' period with unassisted respiration occurred after each ventilation mode to allow the subject to return to a baseline state (figure 1 ).

\section{CMR TECHNIQUE}

All CMR studies were performed using a 1.5 Tesla magnet (Avanto; Siemens Healthineers, Erlangen, Germany). Imaging was performed with retrospective electrocardiographic gating and free-breathing in the fasting state. Vascular flow measurements using PC analysis were accomplished following meticulous slice prescription perpendicular to the target vessel by referencing localiser images at sequential anatomic locations: superior vena cava (SVC), Fontan circuit (for subjects)/suprahepatic inferior vena cava (IVC) (for controls), subhepatic IVC, portal vein (PoV), right/left pulmonary arteries (RPA/LPA), ascending aorta (RPA level) and descending thoracic aorta (diaphragm level). Typical PC parameters included: repetition time ms/echo time ms 5.3/3.34, flip angle $30^{\circ}$, slice thickness $4 \mathrm{~mm}$; number of excitations 2, in-plane spatial resolution $1.3 \times 1.3 \mathrm{~mm}$ and views per segment adjusted to match the true temporal resolution for 25 phases/cardiac cycle. Velocity encoding limits were selected according to vessel of interest and increased in the presence of aliasing. Imaging time was $60-90$ s per PC acquisition. A complete set of flow measurements, at eight target sites using sequential PC prescriptions, were completed at baseline, at each ventilation mode (NPV and BPV) and at study conclusion (return to baseline) (figure 1).

\section{CMR ANALYSIS}

All examinations were performed by a single, dedicated CMR technologist ( $>15$ years CMR experience) and each CMR study was monitored, in its entirety, by two experienced cardiac radiologists (S-JY and GRK (30 and 3 years CMR experience, respectively)) who were responsible for image optimisation and for ongoing quality assurance. All PC analyses were completed using commercially available tools (Q Flow 5.2; Medis Medical Imaging Systems, Leiden, The Netherlands) by a single radiologist blinded to clinical data (GRK) according to previously published methodology (figure 2). ${ }^{14}$ The sum of SVC and Fontan flows were used to represent total PBF given potential for inaccuracies of pulmonary artery flow sampling adjacent to surgical anastomotic sites. In control subjects, PBF was measured as a summation of flows directly sampled at the RPA and LPA. Cardiac output was based on ascending aortic flow. Hepatic venous flow was calculated by subtracting subhepatic IVC flow from suprahepatic IVC flow or Fontan flow.

\section{Safety and tolerability of the external ventilator}

At the conclusion of the CMR study, each participant completed a standardised questionnaire to assess tolerability and preferences for ventilation, blinded to the results of his/her CMR flow measurements. Each statement within the questionnaire

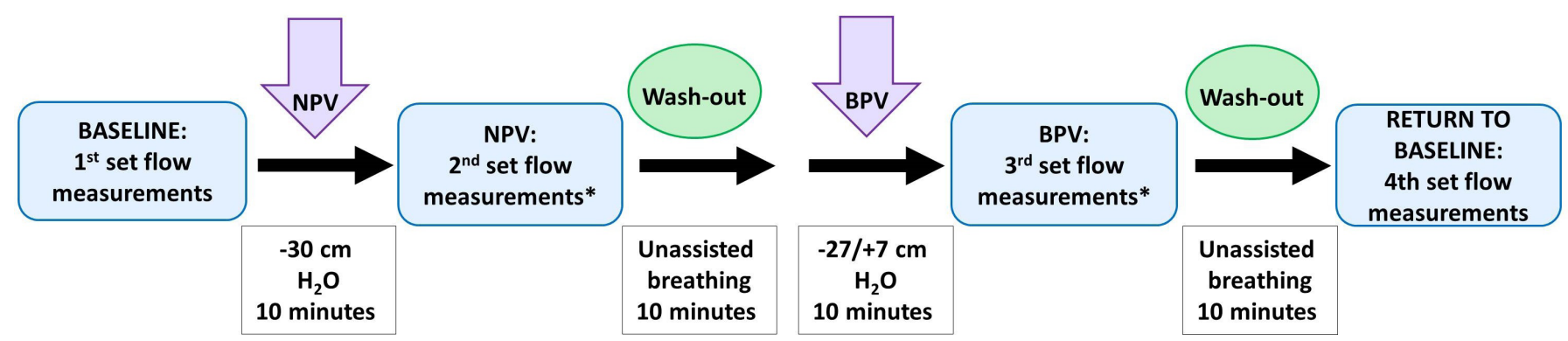

Figure 1 Details of the study protocol. Each set of flow measurements were acquired at the eight target sites (superior vena cava, Fontan pathway/ suprahepatic inferior vena cava, subhepatic inferior vena cava, portal vein, right pulmonary artery, left pulmonary artery, ascending and descending aorta. BPV, biphasic ventilation; NPV, negative pressure ventilation. *Vascular flow measurements completed during ongoing ventilation). 


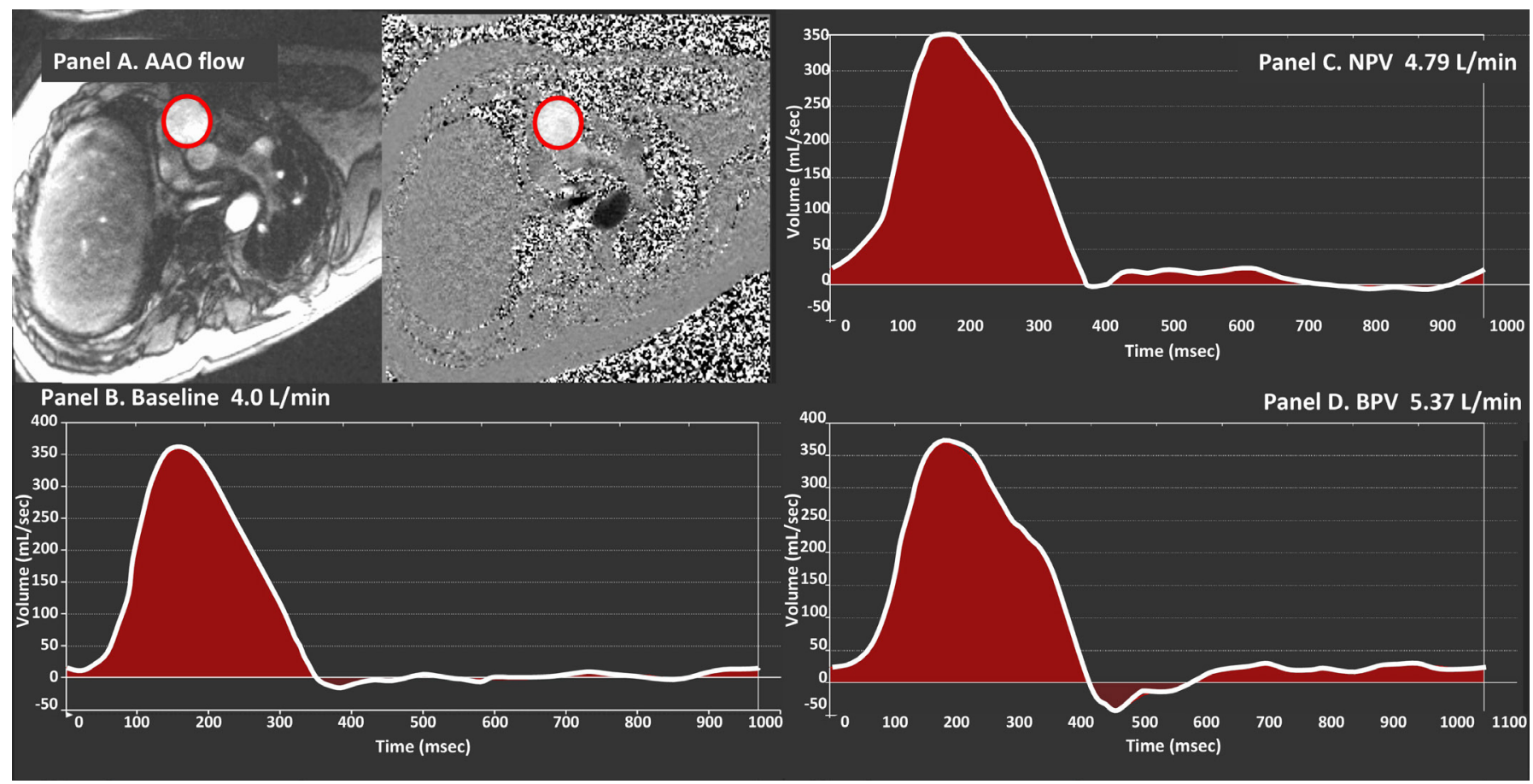

Figure 2 Phase-contrast flow analysis in a Fontan subject measured at the ascending aorta (AAO) (panel A) with a region of interest (shown in red) on the magnitude image (panel $A$, left) and phase image (panel $A$, right). Flow curves at baseline $(4.0 \mathrm{~L} / \mathrm{min}$, panel $B)$, after $10 \mathrm{~min}$ of negative pressure ventilation (NPV) $(4.79 \mathrm{~L} / \mathrm{min}$, panel C) and after $10 \mathrm{~min}$ of biphasic ventilation (BPV) $(5.37 \mathrm{~L} / \mathrm{min}$, panel D).

was associated with a Likert-scale (1-strongly disagree; 2disagree; 3-neutral; 4-agree; 5-strongly agree). Subjects were asked to comment on statements such as 'the cuirass is comfortable to wear', 'the external ventilation device helps me breathe more easily' and "I look forward to using the external ventilation device again”.

\section{STATISTICAL ANALYSIS}

Continuous variables were tested for normality using the Shapiro-Wilk test and were expressed as means (SD) or medians (IQR), as appropriate. Statistical comparisons of continuous variables between groups were made using the Student's t-test or Wilcoxon rank-sum test and within groups using the paired t-test or Wilcoxon signed-rank test. The $\chi^{2}$ test or the Fisher's exact test was applied for categorical variables. Correlations were explored using either the Pearson's or Spearman's correlation coefficient. Change in flow from baseline was assessed using a linear mixed model (ventilation mode, location of flow measurement and group as fixed effects and a random effect for subject). Data were analysed using JMP 13 statistical software package (SAS Institute, Cary, North Carolina, USA). A two-tailed p value $<0.05$ was considered to be statistically significant.

\section{RESULTS}

\section{Patient population}

Between December 2017 and May 2018, 80 Fontan subjects seen in the ambulatory clinic were screened for inclusion. Of these, 15 adults were deemed eligible: 11 adults consented and successfully completed the CMR study and 4 declined enrolment. One patient was excluded following review of CMR flow data due to susceptibility artefact in the Fontan conduit related to an indwelling stent. Therefore, the final cohort consisted of 10 study patients $(50 \%$ male) at median age 24.5 years (IQR 20.8-34.0 years) and 10 matched controls (table 1 ). The two groups did not differ with respect to age, sex or body mass index. Native cardiac anatomy consisted of tricuspid atresia $(n=4)$, double inlet LV $(n=3)$ and hypoplastic right ventricle with pulmonary atresia/stenosis $(n=3)$. Fontan connections were lateral tunnel $(n=6)$ or extracardiac conduit $(n=4)$.

\section{Cardiovascular flow assessment by CMR}

Flow measurements at baseline are shown for Fontan subjects and controls (table 1). Fontan patients had significantly decreased SVC flows and PBF as compared with controls; Fontan circuit flows in subjects were decreased as compared with corresponding suprahepatic IVC flow in controls. Detailed cardiovascular flow measurements during NPV and BPV modes are shown (table 2, figure 3). Statistically significant increases in Fontan flows and PBF occurred during NPV, although to a lesser degree as compared with BPV; the increase in CO during NPV was not statistically significant. Specifically, during NPV, PBF increased by $8 \%\left(\Delta 0.20 \mathrm{~L} / \mathrm{min} / \mathrm{m}^{2}\right.$ (IQR $\left.\left.0.10-0.53\right), \mathrm{p}=0.011\right)$ while CO did not change significantly $(\Delta 0.17(-0.11-0.23)$, $\mathrm{p}=0.432$ ) (table 3 ). In contrast, during BPV, significant augmentation of flows was evident at multiple levels including the Fontan pathway, IVC and hepatic veins as well as an increase in PBF and CO. Notably, BPV increased PBF by $25 \%(\Delta 0.61 \mathrm{~L} /$ $\left.\mathrm{min} / \mathrm{m}^{2}(0.20-0.84), \mathrm{p}=0.002\right)$ and $\mathrm{CO}$ by $16 \%(\Delta 0.47 \mathrm{~L} / \mathrm{min} /$ $\left.\mathrm{m}^{2}(0.21-0.71), \mathrm{p}=0.010\right)$ compared with baseline (table 3 ). The magnitude of impact of NPV and BPV on PBF and CO is shown graphically (figure 4). Application of an identical ventilation protocol in controls did not result in statistically significant augmentation of flows within any of the vessels measured. The linear mixed model demonstrated that least-square mean estimates of change in flow $\left(\mathrm{L} / \mathrm{min} / \mathrm{m}^{2}\right)$ were significantly higher with BPV compared with NPV $(\Delta 0.11$ (95\% CI 0.03 to 0.19$)$ vs $\Delta 0.03(95 \% \mathrm{CI}-0.05$ to 0.11$), \mathrm{p}=0.010)$ and in subjects compared with controls $(\Delta 0.22$ (95\% CI 0.11 to 0.32$)$ vs -0.08 
Table 1 Baseline characteristics of the study population

\begin{tabular}{|c|c|c|c|}
\hline Demographic characteristics & $\begin{array}{l}\text { Fontan subjects } \\
(\mathrm{n}=10)\end{array}$ & $\begin{array}{l}\text { Controls } \\
(n=10)\end{array}$ & $P$ value \\
\hline Age (years) & $24.5(20.8-34.0)$ & $30.5(27.5-43.3)$ & 0.075 \\
\hline Male sex & $5(50)$ & $5(50)$ & $>0.999$ \\
\hline BMI $\left(\mathrm{kg} / \mathrm{m}^{2}\right)$ & $23.0(21.0-27.0)$ & $22.0(20.8-25.0)$ & 0.761 \\
\hline Age at Fontan procedure (years) & $2.0(2.0-7.5)$ & - & - \\
\hline \multicolumn{4}{|l|}{ Indexed CMR flows (L/min $\left./ \mathrm{m}^{2}\right)$} \\
\hline SVC & $0.72(0.55-0.93)$ & $1.05(0.97-1.15)$ & 0.006 \\
\hline Fontan/Sup-IVC & $1.75(1.67-2.07)$ & $2.10(1.97-2.38)$ & 0.031 \\
\hline Sub-IVC & $0.99(0.80-1.37)$ & $1.33(1.17-1.47)$ & 0.049 \\
\hline HV & $0.81(0.62-1.03)$ & $0.79(0.74-1.03)$ & 0.734 \\
\hline PoV & $0.51(0.37-0.60)$ & $0.59(0.51-0.78)$ & 0.104 \\
\hline PBF & $2.48(2.33-2.99)$ & $3.37(3.09-3.81)$ & 0.007 \\
\hline $\mathrm{CO}$ & $2.88(2.28-3.35)$ & $2.97(2.76-3.42)$ & 0.345 \\
\hline \multicolumn{4}{|l|}{ Cardiopulmonary function tests* } \\
\hline Resting oxygen saturation (\%) & $98(90-99)$ & - & - \\
\hline Spirometry & & - & - \\
\hline FEV 1 (absolute, L) & $2.99(2.01-3.49)$ & & \\
\hline $\mathrm{FEV}_{1}(\%$ predicted $)$ & $71(55-79)$ & & \\
\hline FVC (absolute, L) & $3.41(2.34-4.13)$ & & \\
\hline FVC (\% predicted) & $63(56-80)$ & & \\
\hline $\mathrm{FEV}_{1} / \mathrm{FVC}$ (absolute) & $86(81-86)$ & & \\
\hline $\mathrm{FEV}_{1} / \mathrm{FVC}$ (\% predicted) & $100(95-107)$ & & \\
\hline $\mathrm{Peak} \mathrm{VO}_{2}$ & & - & - \\
\hline Absolute (cc/kg/min) & $22.1(17.8-25.0)$ & & \\
\hline$\%$ predicted & $53(46-68)$ & & \\
\hline $\begin{array}{l}\mathrm{VO}_{2} \text { at anaerobic threshold } \\
\text { (\% of peak predicted) }\end{array}$ & $37(34-49)$ & - & - \\
\hline $\mathrm{VE} / \mathrm{VCO}_{2}$ at anaerobic threshold & $34(30-43)$ & - & - \\
\hline Peak RER & $1.07(1.04-1.16)$ & - & - \\
\hline
\end{tabular}

Data expressed as median (IQR) and absolute number (\%).

${ }^{*}$ Cardiopulmonary function tests within 6 months of study entry are shown.

$\mathrm{BMI}$, body mass index; CMR, cardiovascular magnetic resonance; $\mathrm{CO}$, cardiac output; FEV, forced expiratory volume in $1 \mathrm{~s}$; FVC, forced vital capacity; HV, hepatic vein; PBF, pulmonary blood flow; PoV, portal vein; RER, respiratory exchange ratio; Sub-IVC, subhepatic inferior vena cava; Sup-IVC, suprahepatic inferior vena cava; SVC, superior vena cava; $\mathrm{VE} / \mathrm{VCO}_{2}$, ventilatory efficiency; $\mathrm{VO}_{2}$, oxygen consumption.

(95\% CI -0.19 to 0.02 ), $\mathrm{p}<0.001$ ). Flows measured following the 'washout' did not differ from baseline confirming the acute yet transient effects of external ventilation (all $\mathrm{p}>0.05$ ).

\section{SAFETY AND TOLERABILITY}

There were no adverse events or side effects reported by any of the participants and the short-term ventilation trial was welltolerated by all subjects. Questionnaire results revealed that more Fontan patients agreed that the cuirass ventilator was comfortable $(4(4,5)$ vs $3(3,4) ; \mathrm{p}=0.026)$ and a greater number expressed a willingness to wear the device again $(3.5(3,4)$ vs $2.5(1,2.5) ; p=0.001)$ as compared with controls. While all the Fontan patients preferred the BPV mode to the NPV (100\%), the controls were divided in their opinion (40\% preferred BPV and $60 \%$ preferred NPV). There was a significant correlation between those who expressed the greatest desire for repeat ventilation and the extent of increase in $\operatorname{PBF}(r=0.59, \mathrm{p}=0.008)$ and $\mathrm{CO}(\mathrm{r}=0.70, \mathrm{p}=0.001)$, respectively (figure 5).

\section{DISCUSSION}

To our knowledge, this is the first description of the impact of differing external ventilation strategies on CMR flows in ambulatory adult Fontan patients with comparison to healthy controls. The principal, novel findings to emerge from this study include:
1. A short-term, external ventilation strategy resulted in significant augmentation of PBF and CO in Fontan subjects as compared with controls where no significant change was observed.

2. BPV resulted in a greater magnitude and extent of vascular flow increase as compared with NPV.

3. The strength of an individual's desire for continued ventilation correlated with the magnitude of augmented vascular flow.

\section{Negative pressure ventilation in the congenital heart disease population}

The negative pressure mode of ventilation used in contemporary practice is based on principles described during the poliomyelitis epidemics in the 19th century, when the iron lung was used for paralysed patients with respiratory insufficiency. ${ }^{15}$ Subsequently, positive pressure ventilation (PPV) became the more widely applied mode of artificial ventilation, notwithstanding the adverse cardiorespiratory interactions documented by Cournand et al linking intermittent PPV to a decline in CO, a concern particularly magnified in single ventricle physiology. ${ }^{16}$ In the 1990s, interest in NPV was renewed for children with congenital heart disease in the acute care setting. ${ }^{17}$ While PPV was found to be detrimental to cardiac flows, NPV was reported to 
Table 2 Flow measurements at baseline and following external ventilation in Fontan subjects and controls

\begin{tabular}{|c|c|c|c|c|c|}
\hline $\begin{array}{l}\text { CMR flows } \\
\left(\mathrm{L} / \mathrm{min} / \mathrm{m}^{2}\right)\end{array}$ & Baseline & Post-NPV & $P$ value & Post-BPV & $P$ value \\
\hline \multicolumn{6}{|c|}{ Fontan subjects $(n=10)$} \\
\hline SVC & $0.72(0.55-0.93)$ & $0.75(0.59-0.90)$ & 0.652 & $0.80(0.74-1.04)$ & 0.106 \\
\hline Fontan & $1.75(1.67-2.07)$ & $1.99(1.88-2.47)$ & 0.049 & $2.36(1.81-3.02)$ & 0.002 \\
\hline Sub-IVC & $0.99(0.80-1.37)$ & $1.16(0.91-1.48)$ & 0.106 & $1.37(0.88-1.80)$ & 0.027 \\
\hline HV & $0.81(0.62-1.03)$ & $0.96(0.85-1.04)$ & 0.160 & $1.06(0.84-1.42)$ & 0.049 \\
\hline PoV & $0.51(0.37-0.60)$ & $0.53(0.45-0.75)$ & 0.129 & $0.55(0.42-0.60)$ & 0.432 \\
\hline PBF & $2.48(2.33-2.99)$ & $2.88(2.51-3.55)$ & 0.037 & $3.08(2.70-3.76)$ & 0.002 \\
\hline $\mathrm{CO}$ & $2.88(2.28-3.35)$ & $2.82(2.55-3.47)$ & 0.432 & $3.28(2.81-3.72)$ & 0.010 \\
\hline \multicolumn{6}{|c|}{ Controls $(n=10)$} \\
\hline SVC & $1.05(0.97-1.15)$ & $1.10(0.92-1.17)$ & 0.770 & $1.01(0.90-1.15)$ & 0.426 \\
\hline Sup-IVC & $2.10(1.97-2.38)$ & $2.06(1.68-2.30)$ & 0.156 & $2.19(1.77-2.47)$ & 0.557 \\
\hline Sub-IVC & $1.33(1.17-1.47)$ & $1.42(1.18-1.60)$ & 0.770 & $1.39(1.22-1.64)$ & 0.573 \\
\hline HV & $0.79(0.74-1.03)$ & $0.64(0.57-0.96)$ & 0.065 & $0.87(0.72-1.07)$ & 0.570 \\
\hline PoV & $0.59(0.51-0.78)$ & $0.63(0.54-0.78)$ & 0.557 & $0.51(0.48-0.64)$ & 0.275 \\
\hline PBF & $3.37(3.09-3.81)$ & $3.32(2.86-3.68)$ & 0.232 & $2.97(2.64-3.57)$ & 0.084 \\
\hline $\mathrm{CO}$ & $2.97(2.76-3.42)$ & $3.23(2.78-3.43)$ & 0.846 & $3.13(2.76-3.45)$ & 1.000 \\
\hline
\end{tabular}

Data expressed as median (IQR).

Bolded figures are statistically significant to $\mathrm{p}<0.05$

$\mathrm{BPV}$, biphasic ventilation; $\mathrm{CMR}$, cardiovascular magnetic resonance; $\mathrm{CO}$, cardiac output; $\mathrm{HV}$, hepatic vein; NPV, negative pressure ventilation; PBF, pulmonary blood flow; PoV, portal vein; Sub-IVC, subhepatic inferior vena cava; Sup-IVC, suprahepatic inferior vena cava; SVC, superior vena cava.

enhance PBF and CO, particularly in those with single ventricle physiology. ${ }^{101819}$ Shekerdemian et al specifically evaluated the effect of conversion of PPV to NPV in ventilated children after a Fontan operation and demonstrated an acute increase in PBF, which could be further increased with extended therapy. ${ }^{11}$ Unanimously, these studies demonstrated the beneficial role of NPV in children early after surgery for single ventricle palliation but limitations in technology relegated use to the acute care setting.

\section{FLOWS IN THE FONTAN: PBF AND CO}

In line with previous reports, we documented relatively low baseline PBF in the Fontan subjects as compared with matched controls. ${ }^{40}$ The lack of an active ventricular pump to deliver blood to the pulmonary circulation within the Fontan circuit is the main reason for this difference. Limitations in CO in the Fontan population have primarily been attributed to increased pulmonary vascular resistance, ventricular dysfunction, decreased heart rate and/or increased afterload. ${ }^{321} 22$ The respiratory 'pump' will drive pulmonary venous return as a result of decreased intrathoracic pressures during inspiration which results in increased systemic venous return. Findings of poor chest wall compliance, respiratory muscle weakness and restrictive lung physiology, which are commonly observed in the Fontan population, may therefore have deleterious effects on cardiovascular flows. ${ }^{23-25}$ Specifically, insufficient intrathoracic or intra-abdominal pressures during inspiration may in turn result in decreased preload, reduced $\mathrm{PBF}$ and/or decreased CO.

\section{Augmentation of Fontan flows: the role of external ventilation}

Spontaneous inspiration has been shown to enhance PBF and stroke volume across the spectrum of Fontan circulations. ${ }^{26}$ It stands to reason that the application of a non-invasive external thoraco-abdominal ventilator to enhance preload through increased negative intrathoracic pressure in the Fontan patient would be an effective non-invasive way to modulate intracardiac flows with subsequent amelioration in CO. In our study, we documented heart rate stability during the ventilation process and we therefore attributed the improvement in $\mathrm{CO}$ to augmentation of the stroke volume through changes in intrathoracic/ intra-abdominal pressures. ${ }^{11}$

We found that BPV was superior to NPV in the Fontan population, and this aligns well with the potential contributions of both intra-abdominal and intrathoracic pressures on venous return. ${ }^{27}$ We submit that while NPV affects the inspiratory phase of breathing only, relying on passive recoil for exhalation; BPV potentially augments the pressure gradient for venous return throughout the respiratory cycle by effectively reducing the intrathoracic pressure during inspiration as well as increasing intra-abdominal pressure during exhalation. This would minimise retrograde venous flow and augment venous return into the thorax from the abdomen during exhalation-the net effect being a sustained beneficial effect of BPV on venous return and cardiac efficiency throughout the respiratory cycle. ${ }^{28}$

In the context of an absent subpulmonary ventricle, the diaphragm is considered to be the most important inspiratory muscle for maintenance of venous return in the Fontan population. ${ }^{29}$ Accordingly, we observed an increase in Fontan circuit, IVC and HV flows following BPV. The lack of change in PoV flows evident in our population is not an unexpected finding and has been previously documented in the Fontan population (possibly attributable to decreased hepatic compressibility, increased sinusoidal pressure and/or hepatic congestion). ${ }^{30}$

\section{External ventilation: feasibility, safety, tolerability and desirability}

All subjects were able to tolerate the study protocol without adverse effects. The strong correlation between a subject's desire to use the external ventilation device again and the magnitude of effect on PBF and $\mathrm{CO}$ is a notable observation. Further study will be required to explore the benefit of longer duration of ventilation and the relationship between augmentation of cardiovascular flows and objective markers of wellbeing (including measures of quality of life, cardiopulmonary exercise results and incidence of major adverse cardiovascular events). 

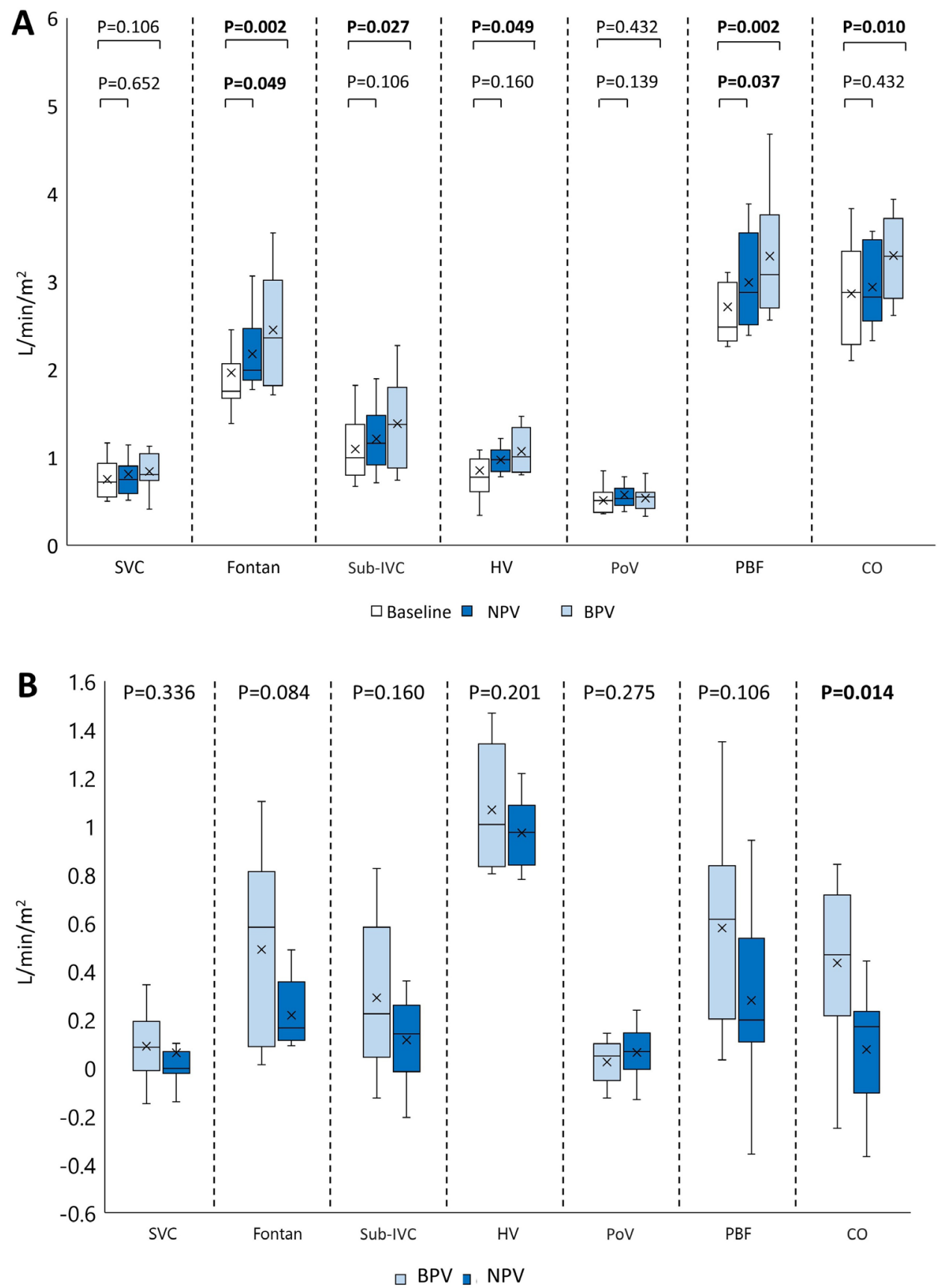

Figure 3 Impact of external ventilation modes on vascular flows are shown in Fontan subjects $(n=10)$. Measurements of indexed flows are shown at baseline and following biphasic ventilation (BPV) and negative pressure ventilation (NPV) (panel A). Magnitude of change according to ventilation mode is also shown (delta BPV and delta NPV) (panel B). CO, cardiac output; HV, hepatic vein; PBF, pulmonary blood flow; PoV, portal vein; Sub-IVC, subhepatic inferior vena cava; SVC, superior vena cava.

\section{Limitations and future directions}

Of mention, our study is limited by measurement of the acute effects of ventilation on vascular flows in a relatively homogeneous patient population, which may therefore restrict generalisability of our results. Investigating sustainability or estimation of the required time for device application to achieve long-term benefit was beyond the scope of this exploratory study. Nevertheless, even if the increases in PBF and CO were restricted to the time of active ventilation, the potential impact could be substantial (for instance, if the external device could be used at home during sleep, in a manner similar to continuous positive airway pressure, the augmentation of PBF by $25 \%$ and CO by $16 \%$ for even a subset of the day would undoubtedly result in improvements in organ perfusion with concomitant benefit). Second, since all CMR examinations were performed in the supine position, the effect of gravity on the systemic venous return in an upright patient undergoing external ventilation was not evaluated. While the CMR suite allowed for comprehensive flow measurements and could be potentially used for future up-titration of therapy, the obligate presence of the magnetic coil between the cuirass and the anterior chest wall inherently prevented direct contact of the ventilator with the patient resulting in uncertain impact on efficiency of ventilation. Additionally, order of the ventilation modes was not randomised and we were unable to definitively exclude the potential additive effect of BPV following 


\begin{tabular}{|c|c|c|c|}
\hline $\begin{array}{l}\text { CMR flows } \\
\Delta \text { flow }\left(\mathrm{L} / \mathrm{min} / \mathrm{m}^{2}\right)\end{array}$ & $\begin{array}{l}\text { Fontan subjects } \\
(\mathrm{n}=10)\end{array}$ & $\begin{array}{l}\text { Controls } \\
(n=10)\end{array}$ & $P$ value \\
\hline \multicolumn{4}{|l|}{ Effect of NPV } \\
\hline SVC & $0.00(-0.02-0.07)$ & $0.04(-0.31-0.23)$ & 0.571 \\
\hline Fontan/Sup-IVC & $0.16(0.11-0.35)$ & $-0.21(0.48-0.05)$ & 0.004 \\
\hline Sub-IVC & $0.14(-0.02-0.26)$ & $0.01(-0.21-0.30)$ & 0.472 \\
\hline HV & $0.14(0.01-0.30)$ & $-0.21(-0.38--0.01)$ & 0.026 \\
\hline PoV & $0.07(-0.01-0.14)$ & $0.02(-0.04-0.10)$ & 0.345 \\
\hline PBF & $0.20(0.10-0.53)$ & $-0.09(-0.43-0.09)$ & 0.009 \\
\hline $\mathrm{CO}$ & $0.17(-0.11-0.23)$ & $0.07(-0.31-0.23)$ & 0.571 \\
\hline \multicolumn{4}{|l|}{ Effect of BPV } \\
\hline SVC & $0.08(-0.01-0.19)$ & $0.02(-0.16-0.09)$ & 0.089 \\
\hline Fontan/Sup-IVC & $0.58(0.09-0.81)$ & $0.00(-0.50-0.15)$ & 0.009 \\
\hline Sub-IVC & $0.22(0.04-0.58)$ & $-0.01(-0.26-0.30)$ & 0.111 \\
\hline HV & $0.26(0.04-0.58)$ & $-0.03(-0.12-0.05)$ & 0.045 \\
\hline PoV & $0.05(-0.05-0.10)$ & $-0.04(-0.12-0.02)$ & 0.088 \\
\hline PBF & $0.61(0.20-0.84)$ & $-0.27(-0.55-0.13)$ & 0.001 \\
\hline $\mathrm{CO}$ & $0.47(0.21-0.71)$ & $0.07(-0.47-0.33)$ & 0.034 \\
\hline
\end{tabular}

Data expressed as median (IQR).

$\mathrm{BPV}$, biphasic ventilation; $\mathrm{CMR}$, cardiovascular magnetic resonance; $\mathrm{CO}$, cardiac output; $\mathrm{HV}$, hepatic vein; NPV, negative pressure ventilation; PBF, pulmonary blood flow; PoV, portal vein; Sub-IVC, subhepatic inferior vena cava; Sup-IVC, suprahepatic inferior vena cava; SVC, superior vena cava.

NPV. However, since the flows measured following the $10 \mathrm{~min}$ washout period were similar to baseline, suggesting the transient effect of external ventilation, we believe that the intervening washout time between ventilation modes was sufficient to allow for re-establishment of a cardiorespiratory steady
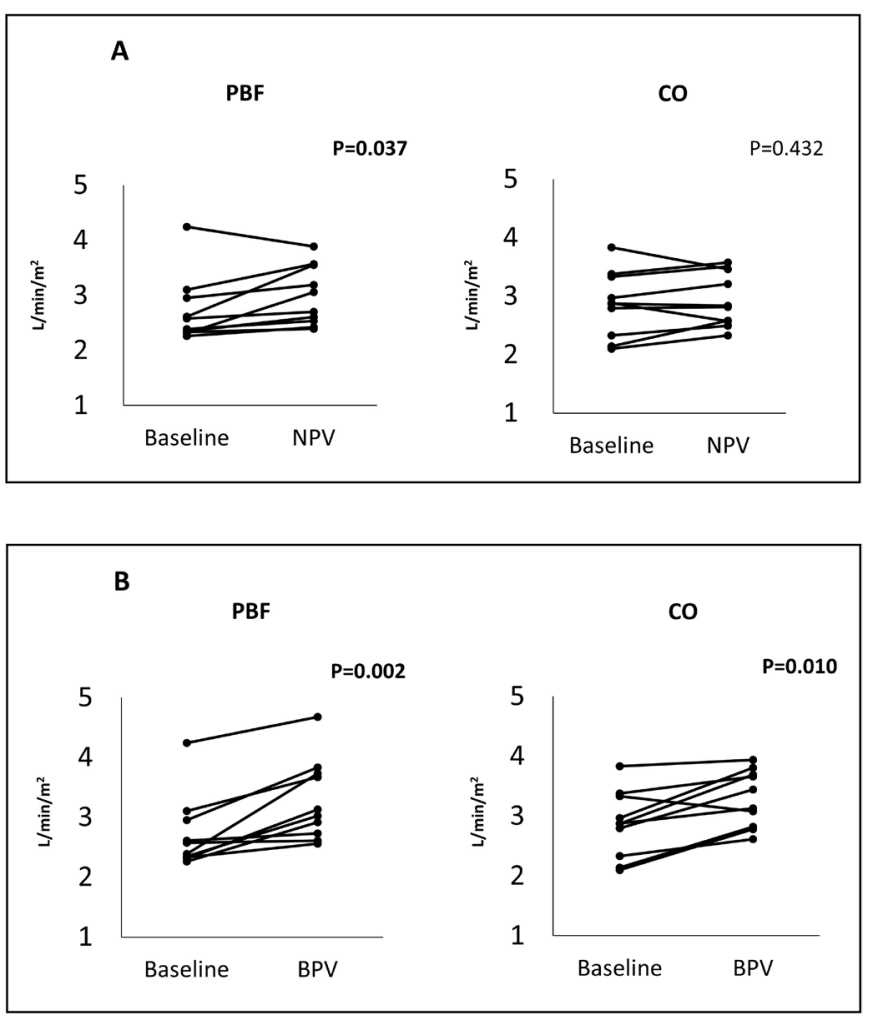

Figure 4 The impact of ventilation modes on pulmonary blood flow (PBF) and cardiac output (CO) in Fontan patients as compared with baseline. Negative pressure ventilation (NPV) (panel A) and biphasic ventilation (BPV) (panel B) are demonstrated.
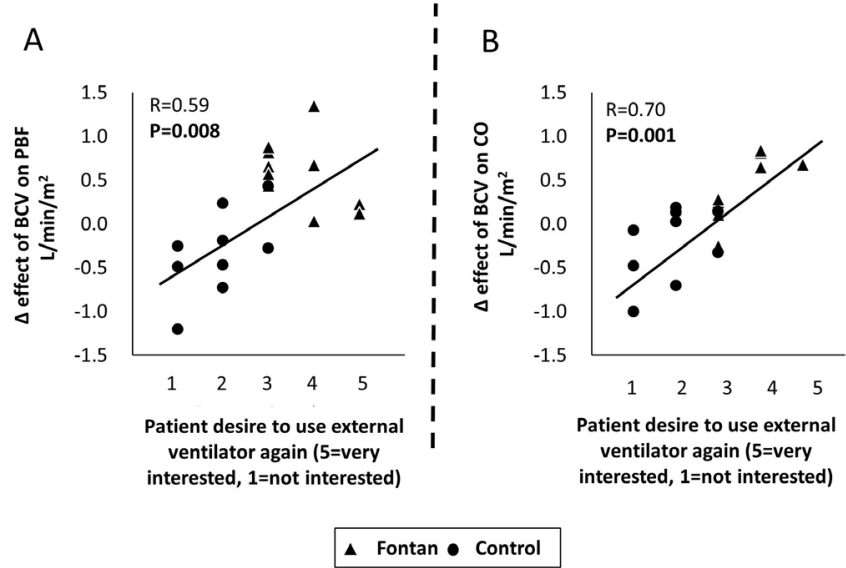

Figure 5 The relationship between subjective tolerability of biphasic ventilation (BPV) and haemodynamic response. Correlations between subjective tolerability and pulmonary blood flow (PBF) (panel A) and cardiac output (CO) (panel B) are shown for all subjects (Fontan patients $(n=10)$ and controls $(n=10))$.

state. Furthermore, the direct assessment of the impact of NPV and BPV on tidal volume, intrathoracic pressures and intraabdominal pressures would be of interest, although beyond the scope of this proof-of-concept study. Lastly, the results of our pilot study should be interpreted with caution in the context of our small sample size, limited inclusion of patients with dominant LV morphologies and only modest study follow-up. The impact of external ventilation in patients with failing Fontan physiology, as a bridge to advanced therapies, or perhaps as destination therapy in and of itself, is a promising avenue worthy of future investigation.

\section{Key questions}

What is already known on this subject?

- Failing Fontan physiology is increasingly being recognised late after Fontan palliation and has been associated with significant morbidity and mortality as a result of low cardiac output (CO).

- Therapeutic options for those with failing Fontan physiology are extremely limited.

\section{What might this study add?}

- A short-term, non-invasive external thoraco-abdominal ventilation strategy is shown to be associated with significant augmentation of pulmonary blood flow (PBF) and $\mathrm{CO}$ as measured by cardiovascular magnetic resonance imaging in Fontan subjects as compared with controls.

- This is the first description of a biphasic ventilation strategy in the Fontan population.

- Biphasic ventilation resulted in a greater magnitude and extent of vascular flow increase as compared with negative pressure ventilation.

How might this impact on clinical practice?

- In the context of limited therapeutic options available for Fontan patients at risk for late complications, non-invasive augmentation of PBF and CO by external ventilation may represent an important step forward in the ambulatory care of this high-risk population. 


\section{CONCLUSION}

Augmentation of PBF and CO can be achieved following application of a short-term external thoraco-abdominal ventilation strategy in adults late after Fontan palliation. The impact of BPV on cardiovascular flow augmentation was superior to the NPV mode and the mechanistic underpinnings of this observation are worthy of future study. These early findings should be confirmed in larger patient cohorts with greater anatomic diversity, longer periods of external ventilation and extended follow-up intervals to determine the sustainability of the haemodynamic effects and the potential impact of external ventilation on clinical outcomes.

Twitter Pradeepkumar Charla @charla789 and Rachel M Wald @DrRachelWald

Contributors PC and RW were responsible for conception, design of the study and obtaining funding. PC, GRK and S-JY contributed to subject recruitment and data acquisition. GRK analysed the CMR studies. KY and KH contributed to statistical analysis. All authors made substantial contributions to analysis and interpretation of data. PC, GRK and RW drafted the manuscript. All authors critically revised and reviewed the manuscript. All authors read and approved the final manuscript. PC, GRK and RW agreed to be responsible for the overall content as guarantors. The corresponding author has the right to grant on behalf of all authors and does grant on behalf of all authors, an exclusive licence (or non-exclusive for government employees) on a worldwide basis to the BMJ Publishing Group Ltd (BMJPGL) and its licensees to permit this article (if accepted) to be published in Heart editions and any other BMJPGL products to exploit all subsidiary rights.

Funding PC and RW were recipients of grant support from the Peter Munk Cardiac Centre Innovation Committee.

Competing interests None declared.

Patient and public involvement Patients and/or the public were not involved in the design, conduct, reporting or dissemination plans of this research.

Patient consent for publication Not required.

Ethics approval This prospective study received approval from institutional research ethics boards (Toronto General Hospital and SickKids) as well as Health Canada.

Provenance and peer review Not commissioned; externally peer reviewed. Data availability statement Data are available on reasonable request.

Open access This is an open access article distributed in accordance with the Creative Commons Attribution Non Commercial (CC BY-NC 4.0) license, which permits others to distribute, remix, adapt, build upon this work non-commercially, and license their derivative works on different terms, provided the original work is properly cited, appropriate credit is given, any changes made indicated, and the use is non-commercial. See: http://creativecommons.org/licenses/by-nc/4.0/.

ORCID iDs

Gauri Rani Karur http://orcid.org/0000-0002-3757-7888

Kenichiro Yamamura http://orcid.org/0000-0003-2876-1023

\section{REFERENCES}

1 Fontan F, Baudet E. Surgical repair of tricuspid atresia. Thorax 1971;26:240-8.

2 Jonas RA, Castaneda AR. Modified Fontan procedure: atrial baffle and systemic venous to pulmonary artery anastomotic techniques. J Card Surg 1988;3:91-6.

3 Pundi KN, Johnson JN, Dearani JA, et al. 40-Year follow-up after the Fontan operation: long-term outcomes of 1,052 patients. J Am Coll Cardiol 2015;66:1700-10.

4 Khairy P, Fernandes SM, Mayer JE, et al. Long-Term survival, modes of death, and predictors of mortality in patients with Fontan surgery. Circulation 2008;117:85-92.
5 d'Udekem Y, lyengar AJ, Galati JC, et al. Redefining expectations of long-term survival after the Fontan procedure: twenty-five years of follow-up from the entire population of Australia and New Zealand. Circulation 2014;130:S32-8.

6 Huddleston CB. The failing Fontan: options for surgical therapy. Pediatr Cardiol 2007;28:472-6.

7 Deal BJ, Jacobs ML. Management of the failing Fontan circulation. Heart 2012;98:1098-104.

8 Williams DB, Kiernan PD, Metke MP, et al. Hemodynamic response to positive end-expiratory pressure following right atrium-pulmonary artery bypass (Fontan procedure). J Thorac Cardiovasc Surg 1984;87:856-61.

9 Penny DJ, Hayek Z, Rawle P, et al. Ventilation with external high frequency oscillation around a negative baseline increases pulmonary blood flow after the Fontan operation. Cardiol Young 1992;2:277-80.

10 Penny DJ, Hayek Z, Redington AN. The effects of positive and negative extrathoracic pressure ventilation on pulmonary blood flow after the total cavopulmonary shunt procedure. Int J Cardiol 1991;30:128-30.

11 Shekerdemian LS, Bush A, Shore DF, et al. Cardiopulmonary interactions after Fontan operations. Circulation 1997;96:3934-42.

12 Nayak KS, Nielsen J-F, Bernstein MA, et al. Cardiovascular magnetic resonance phase contrast imaging. J Cardiovasc Magn Reson 2015;17:71.

13 Fratz S, Chung T, Greil GF, et al. Guidelines and protocols for cardiovascular magnetic resonance in children and adults with congenital heart disease: SCMR expert consensus group on congenital heart disease. J Cardiovasc Magn Reson 2013;15:51.

14 Caro-Dominguez P, Chaturvedi R, Chavhan G, et al. Magnetic resonance imaging assessment of blood flow distribution in fenestrated and completed Fontan circulation with special emphasis on abdominal blood flow. Korean J Radiol 2019;20:1186-94.

15 Mehta S, Hill NS, ventilation N. Noninvasive ventilation. Am J Respir Crit Care Med 2001:163:540-77.

16 Cournand A, Motley HL. Physiological studies of the effects of intermittent positive pressure. Am J Physiol 1948;152:162-74.

17 Shekerdemian LS, Bush A, Lincoln C, et al. Cardiopulmonary interactions in healthy children and children after simple cardiac surgery: the effects of positive and negative pressure ventilation. Heart 1997;78:587-93.

18 Henneveld HT, Sreeram N, Hitchcock JF. Competitive pulmonary flow in infancy: the effect of respiration. Int J Cardiol 1999;68:9-12.

19 Pierce JM, Jenkins IA, Noyes JP, et al. The successful use of continuous negative extrathoracic pressure in a child with Glenn shunt and respiratory failure. Intensive Care Med 1995;21:766-8

20 Goldberg DJ, Shaddy RE, Ravishankar C, et al. The failing Fontan: etiology, diagnosis and management. Expert Rev Cardiovasc Ther 2011;9:785-93.

21 Averin K, Hirsch R, Seckeler MD, et al. Diagnosis of occult diastolic dysfunction late after the Fontan procedure using a rapid volume expansion technique. Heart 2016:102:1109-14.

22 Gewillig M, Brown SC, Eyskens B, et al. The Fontan circulation: who controls cardiac output? Interact Cardiovasc Thorac Surg 2010;10:428-33.

23 Matthews IL, Fredriksen PM, Bjørnstad PG, et al. Reduced pulmonary function in children with the Fontan circulation affects their exercise capacity. Cardiol Young 2006:16:261-7.

24 Opotowsky AR, Landzberg MJ, Earing MG, et al. Abnormal spirometry after the Fontan procedure is common and associated with impaired aerobic capacity. Am J Physiol Heart Circ Physiol 2014;307:H110-7.

25 Gewillig M, Brown SC. The Fontan circulation after 45 years: update in physiology. Heart 2016;102:1081-6.

26 Penny DJ, Redington AN. Doppler echocardiographic evaluation of pulmonary blood flow after the Fontan operation: the role of the lungs. Br Heart J 1991;66:372-4.

27 van den Berg PCM, Jansen JRC, Pinsky MR. Effect of positive pressure on venous return in volume-loaded cardiac surgical patients. J Appl Physiol 2002;92:1223-31.

28 Shekerdemian L, Bohn D. Cardiovascular effects of mechanical ventilation. Arch Dis Child 1999:80:475-80.

29 Hsia T-Y, Khambadkone S, Bradley SM, et al. Subdiaphragmatic venous hemodynamics in patients with biventricular and Fontan circulation after diaphragm plication. J Thorac Cardiovasc Surg 2007;134:1397-405

30 Hsia TY, Khambadkone S, Redington AN, et al. Effects of respiration and gravity on infradiaphragmatic venous flow in normal and Fontan patients. Circulation 2000;102:11I-148-53. 